\title{
Growth of $\mathrm{Cr}$ on $\mathrm{Cu}(001)$ studied by scanning tunneling microscopy
}

\author{
J. F. Lawler, R. G. P. van der Kraan, and H. van Kempen \\ Research Institute for Materials, University of Nijmegen, Toernooiveld 1, 6525 ED Nijmegen, The Netherlands \\ A. J. Quinn \\ Physics Department, Trinity College, Dublin 2, Ireland \\ (Received 7 July 1995; revised manuscript received 27 November 1995)
}

\begin{abstract}
The growth of ultrathin films of $\mathrm{Cr}$ on $\mathrm{Cu}(001)$ over the temperature range $285-575 \mathrm{~K}$ is reported. Film formation has been studied by scanning tunneling microscopy, Auger electron spectroscopy, and low-energy electron diffraction. The growth is found to be three dimensional in character with the formation of irregular multilayer high islands. The deposited chromium becomes more particulate and bulklike in nature with increasing deposition temperature, with the formation of high surface outgrowths. The films are metastable and agglomerate upon annealing at higher temperatures.
\end{abstract}

\section{INTRODUCTION}

The development of scanning probe microscopies has opened up new horizons for the study of surfaces and interfaces of materials. The field of surface magnetism is just one example where these new tools, coupled with the existing surface analysis techniques, have brought about an increase in interest and activity. Contiguous to this is the advent of more powerful computational facilities, which have been very successful in the calculation and prediction of new magnetic materials and other unusual phenomena. ${ }^{1}$

$\mathrm{Cr}$ represents an interesting case since in its bulk form, though it is only a weak antiferromagnet, it possesses a halffilled $3 d$ shell. The prospect of somehow stabilizing a high spin configuration yields the possibility of forming a large local moment. ${ }^{2,3}$ There are also important similarities to be drawn between $\mathrm{Cr}$ and $\mathrm{Fe}$. Both have a body-centered-cubic (bcc) structure in their equilibrium room-temperature form. They have practically the same lattice parameters: 0.288 and $0.287 \mathrm{~nm}$, respectively. Iron has been shown to grow on $\mathrm{Cu}(001)$ in metastable ultrathin films $(\approx 11$ monolayers $)$ with a face-centered-cubic (fcc) form. ${ }^{4,5}$ Calculations also predict that the fcc $\mathrm{Cr}$ lattice constant would be within $1 \%$ of that of $\mathrm{Cu}^{6}$

The growth of high-quality epitaxial thin films of $\mathrm{Cr}$ is also of interest because of the continued controversy about its surface magnetic properties. It has been proposed ${ }^{7}$ that $\mathrm{Cr}$ possesses an enhanced layered antiferromagnetic structure. In this scheme there is a sequence of (001) layers with alternating magnetization with the surface layer having a magnetic moment 3-4 times higher than in the bulk. This resulted in considerable effort to the determination of the surface magnetization of $\mathrm{Cr}$. There is disagreement between the results of different measurement techniques as to whether an enhanced antiferromagnetic surface exists. ${ }^{8,9}$ Spinpolarized scanning electron microscope images of $\mathrm{Cr}$ wedges grown on iron ${ }^{10}$ have shown oscillations in the in-plane magnetization between successive atomic $\mathrm{Cr}$ layers, but are unable to determine values of it. Scanning tunneling microscopy (STM) measurements ${ }^{11}$ have reported the observation of a change in polarization of the tunnel current when going from one step to the next. This manifested itself as an effective periodic variation in the measured monatomic step height. One of the difficulties with this experiment was the cleaning of the $\mathrm{Cr}$ single crystal surface, a process that took several months. The growth of epitaxial $\mathrm{Cr}$ films with suitable orientation would greatly facilitate further study of this phenomenon.

The growth of $\mathrm{Cr}$ on $\mathrm{Cu}(001)$ has already been studied by a number of groups. An initial low-energy electron diffraction (LEED) investigation ${ }^{12}$ of the system observed two orthogonal domains, with an apparently $3 \times 1$ structure visible for deposited thicknesses greater than 3 monolayer equivalents (MLE). One monolayer equivalent is assumed to be the amount of Cr necessary to form a single bcc layer on the copper surface. Magneto-optic Kerr effect measurements showed no evidence of any ferromagnetic behavior. Inverse photoemission measurements ${ }^{13}$ for the deposition of $\mathrm{Cr}$ on $\mathrm{Cu}(001)$ showed little or no magnetic splitting, though similar measurements on $\mathrm{Cr} / \mathrm{Ag}(001)$ and $\mathrm{Cr} / \mathrm{Au}(001)$ indicated the presence of a large magnetic moment. It was judged from this work that the results obtained for $\mathrm{Cr}$ deposited on $\mathrm{Ag}(001)$ and $\mathrm{Au}(001)$ were antiferromagnetic in origin due to fcc growth, while $\mathrm{Cr}$ on $\mathrm{Cu}(001)$ was considered to be bcc and paramagnetic. LEED, $\mathrm{x}$-ray photoemission spectroscopy, and $\mathrm{x}$-ray photoelectron diffraction studies ${ }^{14,15}$ and the full dynamical LEED study ${ }^{16}$ showed that $\mathrm{Cr}$ retains its bulk body-centered-cubic structure and grows in a multilayer fashion with four equivalent domain orientations. We report here the deposition of submonolayer $\mathrm{Cr}$ films on $\mathrm{Cu}(001)$ and the study of their initial growth stages as a function of temperature by STM, LEED, and Auger electron spectroscopy (AES) in the temperature range $285-575 \mathrm{~K}$.

\section{EXPERIMENT}

The experiments took place under UHV conditions in a system that has been described previously. ${ }^{17}$ The substrate was a mechanically polished $\mathrm{Cu}(001)$ single crystal, with a miscut of less than $0.5^{\circ}$. The surface was cleaned by repeated cycles of Ar ion bombardment $[1 \mathrm{keV}$ at $620 \mathrm{~K}$ (Refs. 17 and 18)] and annealing at $800 \mathrm{~K}$. The substrate 
heater uses an indirect electron beam, which impinges on the back of the sample holder. Prior to deposition the substrate was given a final ion etching and then allowed to cool radiatively from $620 \mathrm{~K}$ to the required deposition temperature, in order to anneal out any remaining surface damage. The cleaned $\mathrm{Cu}(001)$ crystal exhibited a clearly defined $(1 \times 1)$ LEED pattern and impurity levels were below the detection limit of the AES. The surface consisted of a series of broad terraces $(\approx 400 \mathrm{~nm})$ separated by monatomic steps or clusters of steps. On the terraces atomic resolution of the copper surface was clearly evident.

The $\mathrm{Cr}$ (99.995\%) was sublimed from a Knudsen cell held at $1423 \pm 0.5 \mathrm{~K}$ providing a stable deposition rate of 0.25 MLE/min. This was calibrated using a quartz crystal microbalence as well as with AES data, which are in good agreement with the estimates of the island surface coverage determined from the STM images.

\section{RESULTS AND DISCUSSION}

Films of $\mathrm{Cr}$ on $\mathrm{Cu}(001)$ were grown for nominal thicknesses of typically less than 1 MLE and for substrate temperatures between 285 and $573 \mathrm{~K}$. Thicker films $(\approx 3.0$ MLE) were also deposited at room temperature in order to investigate the evolution of the growth. The STM and LEED results will be discussed in relation to the conclusions of other studies of this system using other techniques.

LEED patterns of all the films showed only the presence of a $(1 \times 1)$ surface structure. We ascribe these to the $\mathrm{Cu}(001)$ reflections as the spots become weaker with increased $\mathrm{Cr}$ coverage and were accompanied by the emergence of a diffuse background. This is indicative of a highly disordered surface structure. The fact that no obvious reflections from the $\mathrm{Cr}$ are visible up to thicknesses of $3 \mathrm{MLE}$ is in contrast to other studies of this system. ${ }^{16,14,15}$

The work of Refs. 14 and 15 conclude that $\mathrm{Cr}$ grows with its bulk (bcc) lattice parameters for thicknesses greater than 3 MLE. For lower coverage this lattice is distorted from its bulk parameters to produce epitaxy, i.e., pseudomorphic growth. There are four equivalent domain orientations, aligned with the (110) plane parallel to the $\mathrm{Cu}$ (001) surface and the $\operatorname{Cr}[1 \overline{11}]$ direction parallel to the $\mathrm{Cu}[110]$. Similar LEED patterns have been observed ${ }^{16}$ for thicker films annealed at temperatures up to $570 \mathrm{~K}$. The low coverage films in our study are probably insufficiently thick to obtain this LEED pattern. However, we also only observe the $1 \times 1$ $\mathrm{Cu}(001)$ reflections in our thicker films (3.0 MLE), unlike the other studies ${ }^{16,14,15}$ that show the four domain LEED pattern at these deposition levels. The question of deposition temperature and its effect on film morphology must be considered when discussing these results and this will be done later in conjunction with the STM data.

We present here a series of images of the $\mathrm{Cu}(001)$ surface after the deposition of $\mathrm{Cr}$ over the temperature range 285$575 \mathrm{~K}$. These are chosen to show the variation in topography for similar amounts of $\mathrm{Cr}$ deposited with increasing temperature. Figures 1(a) and 1(b) display STM images of the $\mathrm{Cu}(001)$ surface after deposition of 0.6 and 3.0 MLE of $\mathrm{Cr}$ respectively at $285 \mathrm{~K}$. In both images the growth of irregular multilayer islands is clearly evident. The size of the islands varies over a large range $(2-15 \mathrm{~nm})$. Even with only 0.6

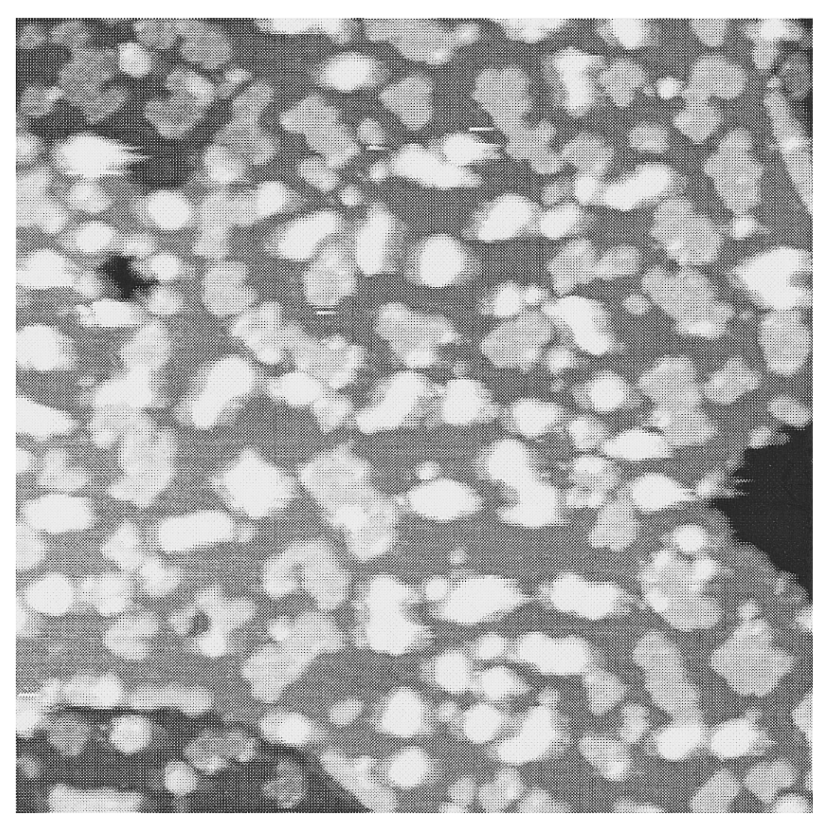

(a)

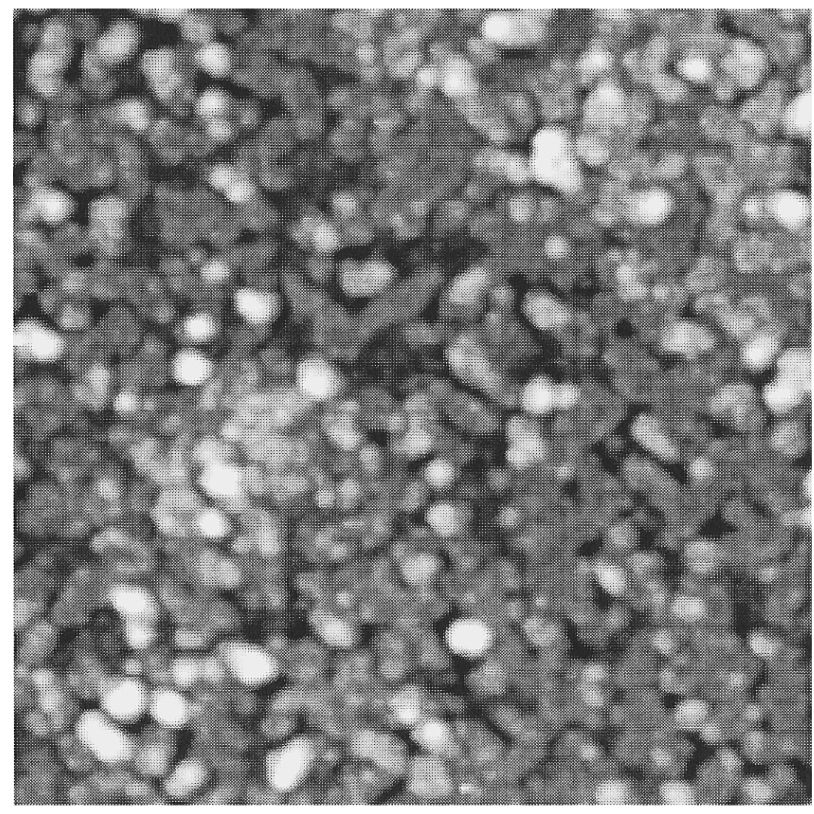

(b)

FIG. 1. STM images of the $\mathrm{Cu}(001)$ surface after deposition of $\mathrm{Cr}$ at $285 \mathrm{~K}\left(\right.$ a) $0.6 \operatorname{MLE}\left(100 \times 100 \times 0.8 \mathrm{~nm}^{3}\right)\left(V_{t}=1.5 \mathrm{~V}\right.$, $\left.I_{t}=100 \mathrm{pA}\right)$ and (b) $3.0 \operatorname{MLE}\left(100 \times 100 \times 0.9 \mathrm{~nm}^{3}\right)\left(V_{t}=10.0 \mathrm{~V}\right.$, $\left.I_{t}=110 \mathrm{pA}\right)$. The islands are irregular and multilayer and vary greatly in size $(2-15 \mathrm{~nm})$. The step heights are characteristic of the $\mathrm{Cr}(110)$ interlayer spacing; there are also some similar to the predicted fcc $\mathrm{Cr}(001)$ spacing. The lack of atomic resolution on the islands is indicative of surface disorder.

MLE deposited [Fig. 1(a)] three-layer-high islands are observed. The copper terrace is clearly visible between the islands at these lower coverages. The STM image in Fig. 2 shows a small-scale atomically resolved image of the $\mathrm{Cu}$ terrace. The measured corrugation on the $\mathrm{Cu}(001)$ surface was as up to $0.6 \mathrm{~nm}$. From the beam widths of their LEED pattern Ref. 14 estimates the size of the islands to be about 


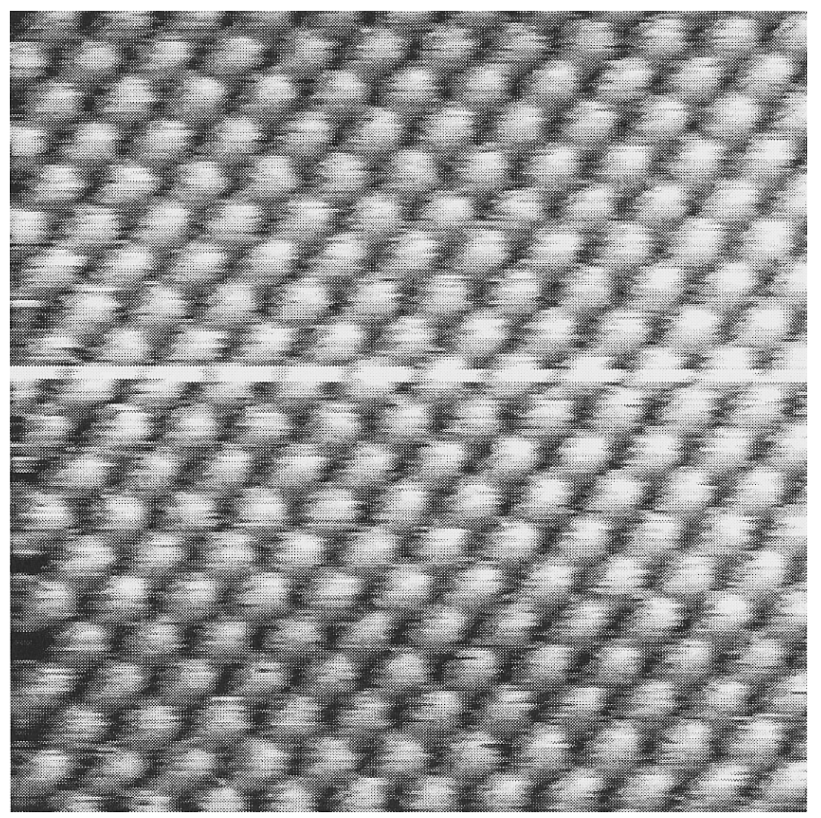

FIG. 2. Small-scale STM images of the $\mathrm{Cu}(001)$ terrace between the $\mathrm{Cr}$ islands $\left(4.3 \times 4.3 \times 0.13 \mathrm{~nm}^{3}\right)$. The atomically resolved images had measured corrugations up to $0.06 \mathrm{~nm}$ high. While it was possible to obtain atomic resolution on the $\mathrm{Cu}$ surface, this was not the case on any of the islands. ( $V_{t}=110 \mathrm{mV}, I_{t}=474 \mathrm{pA}$.)

ten lattice spacings. This is an order of magnitude smaller than those that we observe. Highly disordered islands may be responsible for this anomalous value, implying that they consist of segregated small ordered regions $(\approx 2 \mathrm{~nm})$.

A large spread in step heights was measured from the STM images of films grown at $285 \mathrm{~K}$ [Fig. 1(a)]. Nevertheless, two characteristic island step heights could be determined and these correspond closely to that for the bcc $\mathrm{Cr}(110)$ interlayer spacing $(0.204 \mathrm{~nm})$ and the predicted fcc $\mathrm{Cr}(001)$ spacing $(0.180 \mathrm{~nm})$. The occurrence of this latter spacing was only for a minority of one-monolayer islands. The height histogram in Fig. 3 is of several monolayer-high $\mathrm{Cr}$ islands on the $\mathrm{Cu}(001)$ surface shown in Fig. 1(a). The large peak is the $\mathrm{Cu}$ surface and the $\mathrm{Cr}$ islands while having

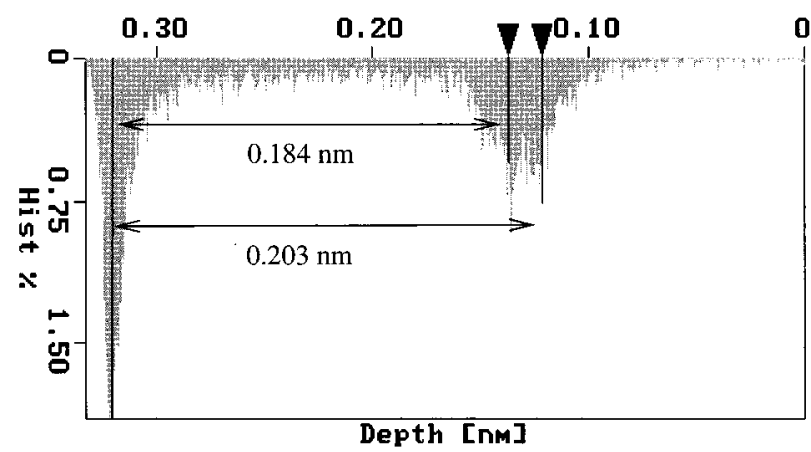

FIG. 3. Height histogram of a several monolayer-high islands from Fig. 1(a). The large peak on the left represents the $\mathrm{Cu}$ surface. Though there is a spread in the step heights of the islands, there appear to be two distinct components at heights of $\sim 0.18 \mathrm{~nm}$ and $\sim 0.20 \mathrm{~nm}$ above the surface.

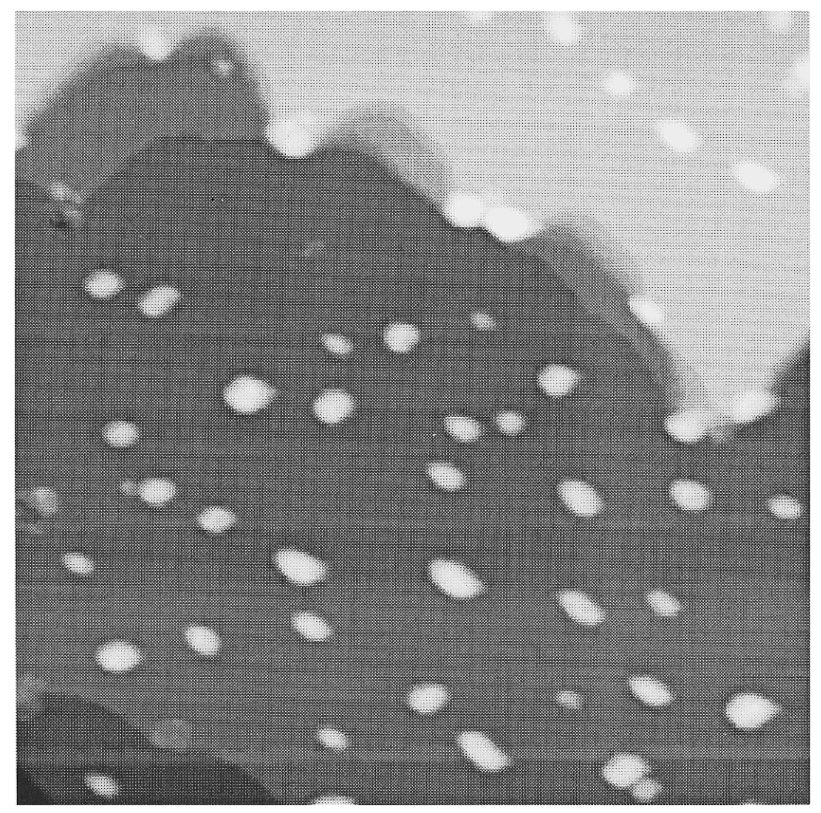

FIG. 4. STM image $\left(200 \times 200 \times 2.5 \mathrm{~nm}^{3}\right)$ after deposition of $0.55 \mathrm{MLE} \mathrm{Cr}$ at $425 \mathrm{~K}$ on the $\mathrm{Cu}(001)$ surface. The islands are typically 4-8 monolayers high and more regular in form than on films grown at lower temperatures. Interlayer mass transport must increase for the islands to grow to these heights. $\left(V_{t}=4.3 \mathrm{~V}\right.$, $I_{t}=100 \mathrm{pA}$.)

a spread in step heights appear to have two distinct components at heights of $\sim 0.18 \mathrm{~nm}$ and $\sim 0.20 \mathrm{~nm}$ above the surface. The layer spacing of $\sim 0.20 \mathrm{~nm}$ supports the assignment of the (110) plane growth parallel to $\mathrm{Cu}(001),{ }^{14,15}$ but the inability to obtain atomic resolution does not allow any confirmation of an epitaxial relationship. Determination of actual heights above the terrace is difficult because in contrast to the flat copper surface where atomic resolution was readily obtained, we were unable to image individual atoms on the islands. The surfaces of the islands appear somewhat irregular even at the nominally one-atomic-step height and in particular the edges are quite indistinct. The blurred $\mathrm{Cr}$ surface could be due to movement of atoms in the islands. Another explanation could be a small contamination of the $\mathrm{Cr}$ surface changing the tip while scanning over the surface or it could be an intrinsic property of the chromium surface as none of the STM reports on chromium have shown atomic resolution. ${ }^{11,19}$

Figures 4 and 5 show the effect of depositing at elevated temperature, $425 \mathrm{~K}$ and $575 \mathrm{~K}$, respectively. The most marked change between these images and the films grown at $285 \mathrm{~K}$ is the reduced number of islands and the increase in their size and height. The higher temperatures augment this process. This produces a reduced effective coverage for similar amounts of material deposited. The AES data also show a large decrease in the $\mathrm{Cr}$ signal $(529 \mathrm{eV})$ for this higher temperature deposition. Films deposited at $285 \mathrm{~K}$ and subsequently annealed at elevated temperature also showed a reduction in their AES signal. $\mathrm{Cr}$ is immiscible in $\mathrm{Cu}$ at these temperatures, so the signal reduction must be due to a decrease in the coverage of the substrate surface. This is confirmed by the STM images, which show the agglomeration of the islands that appear similar in form to those pro- 


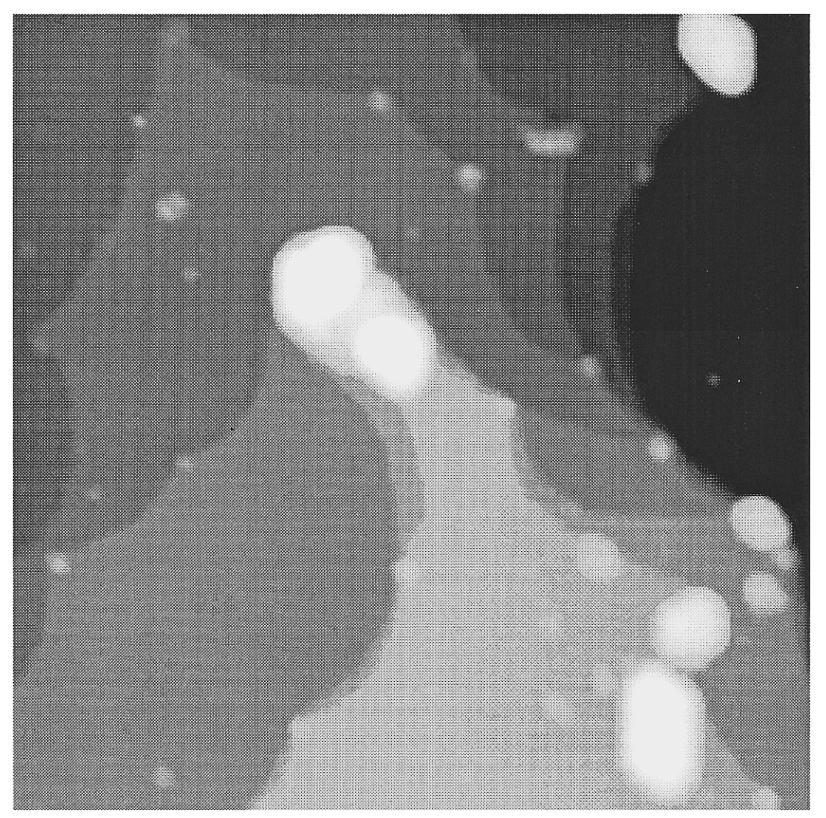

FIG. 5. STM image of the $\mathrm{Cu}(001)$ surface $(200 \times 200 \times 1.5$ $\mathrm{nm}^{3}$ ) after deposition of $0.5 \mathrm{MLE} \mathrm{Cr}$ at $575 \mathrm{~K}$. The large islands tend to form at the apices of step bunches on the $\mathrm{Cu}$ surface. This may be due to diffusion of $\mathrm{Cr}$ along the step edges and their agglomeration in these positions. ( $V_{t}=10.0 \mathrm{~V}, I_{t}=137 \mathrm{pA}$.)

duced by depositing at higher temperature.

The presence of monolayer-high islands with a step height of $\sim 0.18 \mathrm{~nm}$ in the films grown at $285 \mathrm{~K}$ and also at $425 \mathrm{~K}$ is surprising. In both cases their numbers are few and at the higher temperature the rest of the $\mathrm{Cr}$ has agglomerated into particulates. The reason why these monolayer islands are stable and the similarity of their height to the interlayer spacing of $\mathrm{Cu}$ or that predicted for fcc $\mathrm{Cr}$ are unclear. The lack of atomic resolution would seem to preclude the possibility that they are $\mathrm{Cu}$ adislands, so other explanations such as alloying may be considered.

Figure 5 shows a 0.5 -MLE film deposited at $575 \mathrm{~K}$. The surface has a small number of large outgrowths. These are positioned close to the apex of step bunches on the $\mathrm{Cu}$ substrate. There are other particulates on the surface, but these are much smaller when compared with the film deposited at $425 \mathrm{~K}$. There is also evidence, in small-scale images, of slight protrusions at the $\mathrm{Cu}$ step edges. These may be due to the presence of $\mathrm{Cr}$ at the step edge. If this is the case, then it may explain the preferential growth of the large grains at these positions on the surface. The $\mathrm{Cr}$ adatoms could diffuse to these step edges and then travel along them and subsequently agglomerate at the apex of the step bunches and form the larger particulates. The fact that on the film grown at $425 \mathrm{~K}$ the islands next to the step edges are generally larger than those on the terrace would support this hypothesis.

From the STM data it is clear that the growth is by no means layer by layer, as had been originally suggested, ${ }^{12}$ and appears to be better described by a three-dimensional growth system. Reference 15 observed the evolution of the $\mathrm{Cu}(001)$ Tamm state as a function of increased deposition to determine the surface coverage. Their results show a lower surface coverage than would be expected from a random depo- sition model. ${ }^{20}$ In this type of growth the arriving condensate atoms are able to stick anywhere on the surface regardless of the previous topography (hit and stick). They interpret this smaller than expected coverage as evidence of significant mobility and surface diffusion of the $\mathrm{Cr}$ on the $\mathrm{Cu}(001)$ at room temperature. This results in a more particulate, threedimensional growth of the Cr. Our STM images display higher island coverage than these estimates using the Tamm state. For our case of 3.0 MLE deposited we see 95\% coverage of the $\mathrm{Cu}$ surface, while Tamm state measurements give $80 \%$. There is a more marked difference in the submonolayer range: the values at 0.6 MLE are 50\% and 30\%, respectively. The amounts derived from the STM bear a closer relation to the random deposition model. One possible explanation for this disparity is if the films are deposited at different temperatures. When films deposited at $285 \mathrm{~K}$ were annealed to roughly $500 \mathrm{~K}$, we observed a dramatic clustering of the $\mathrm{Cr}$ islands to form particulates. A similar effect is achieved by depositing at elevated temperature; Fig. 4 shows a film of 0.5 MLE deposited at $425 \mathrm{~K}$. So even though both sets of films are deposited nominally at room temperature, a slightly higher deposition temperature in the case of Ref. 15 could bring about this apparent difference in the two measurements. The lack of a distinct LEED diffraction pattern for our $\mathrm{Cr}$ films is further evidence of lower temperature growth, as the full dynamical LEED study ${ }^{16}$ obtained the sharpest diffraction patterns for higher deposition temperatures or after annealing the films.

One problem with this argument is the size of the islands observed by the STM. For the case of the random deposition model a high nucleation density and many small islands are expected. Clearly this is not the situation shown in the STM images. This discrepancy is explained if we consider that there is a relatively high surface diffusivity upon the $\mathrm{Cu}$ terrace and on the $\mathrm{Cr}$ islands, but that interlayer mass transport is negligible at $285 \mathrm{~K}$. Therefore the $\mathrm{Cr}$ forms larger islands, but the overall coverage resembles the random deposition model. As the shape of the islands is irregular the diffusion of $\mathrm{Cr}$ along the island edges should be small. No diffusion along the island edges would lead to the formation of dendritic islands, while a high diffusion rate produces more compact islands. ${ }^{21}$ The type of growth displayed at $285 \mathrm{~K}$ is an intermediary case between fractal and compact island formation. The transition between these two kinds is the source of some disagreement in the literature and has been the subject of recent theoretical calculations. ${ }^{22}$

An argument against a very low interlayer mass transport is given by the observed height distribution. Figure 1(a) shows a large number of monolayer high islands, but also many islands with an almost completed second layer. This distribution should be very different in the case of random deposition and negligible interlayer mass transport. The process responsible for this discrepancy is unclear. A possible explanation is that the development of a second layer on top of a fcc first layer is unstable, while it is favored on top of a bcc $\mathrm{Cr}(110)$ oriented islands. These growth characteristics of monolayer and bilayer island formation at $285 \mathrm{~K}$ are similar to the case of $\mathrm{Cr}$ deposition on $\mathrm{Ag}(001){ }^{23} \mathrm{The} \mathrm{Cr} / \mathrm{Ag}(001)$ system also exhibits analogous behavior upon annealing or high-temperature deposition, though it is reported to have an antiferromagnetically driven stabilization of layer-by-layer 
growth in a narrow temperature and deposition range. The particulate character of films grown at higher temperature (see Figs. 2 and 3) and the agglomeration of annealed films also support the hypothesis of a non-negligible mobility. However, in both these cases interlayer mass transport must become more significant than in the $285 \mathrm{~K}$ case. The film shown in Fig. 4 has a similar amount of $\mathrm{Cr}$ deposited $(0.55$ MLE) as the $285 \mathrm{~K}$ film, but has a lower island density. The heights of these islands are much greater, typically 4-8 monolayers, and are less irregular than those deposited at lower temperatures. Interlayer mass transport therefore must increases at higher temperatures for these higher particulates to form.

These growth characteristics may explain some of the effects observed by Ref. 16 in their LEED patterns. Their sharpest LEED patterns are achieved for films deposited at low temperature and annealed at $520 \mathrm{~K}$. They attribute a reduction in their $\mathrm{Cr}$ AES signal upon annealing to the formation of a $\mathrm{Cu}$ overlayer on top of their $\mathrm{Cr}$ film. The formation of a floating top layer of substrate atoms can occur when the deposited material has a significantly higher surface free energy, as is the case here. Such a difference also tends to preclude wetting of the surface. The creation of a monatomic $\mathrm{Au}$ top layer during the deposition of Fe on $\mathrm{Au}(001)$ (Ref. $24)$ is one example of this. Our STM observations suggest that the reduction in their AES signal on annealing the films is most likely due to the agglomeration of the $\mathrm{Cr}$. The deposition of their films at low temperature $(200 \mathrm{~K})$ should produce a high nucleation density due to the lower surface mobility, close to an ideal random deposition model. The subsequent annealing will form a surface covered with many small particulates. In contrast, deposition at higher temperature produces a surface with fewer growth centers but larger grains. This is because the arriving $\mathrm{Cr}$ atoms have a high surface diffusion and can travel larger distances across the $\mathrm{Cu}$ terrace before they encounter an island.

\section{CONCLUSION}

The growth and surface morphology of $\mathrm{Cr}$ deposited on $\mathrm{Cu}(001)$ have been studied as a function of temperature in the range $285-575 \mathrm{~K}$. At $285 \mathrm{~K}$ the growth is by the formation of irregular multilayer islands. No atomic resolution has been obtained on the $\mathrm{Cr}$, in contrast to the $\mathrm{Cu}$ terrace. The similarity of the surface coverage to the random deposition model suggests that interlayer mass transport remains negligible. However, this is not supported by the observed height distribution. Two distinct island step heights are measured: one similar to the bulk bcc $\mathrm{Cr}(110)$ plane separation, in agreement with the previous studies of this system, and the other can be ascribed to fcc $\mathrm{Cr}(001)$ steps. At higher deposition temperatures the $\mathrm{Cr}$ forms more particulate features due to an increase in interlayer mass transport. The nucleation density is also reduced at elevated growth temperatures due to larger diffusion rates. Annealing the films causes the agglomeration of the films to become more particulate in form.

\section{ACKNOWLEDGMENTS}

The work described here has been financially supported by the Dutch Foundation for Fundamental Research of Matter, which is, in turn, financially supported by the Dutch Organization for Scientific Research. One of us (J.F.L.) would like to acknowledge the assistance of the Human Capital and Mobility program of the European Community, as would A.J.Q. for the support under the same scheme during his stay at the Nijmegen laboratory.
${ }^{1}$ A.J. Freeman and Ru-qian Wu, J. Magn. Magn. Mater. 100, 497 (1991).

${ }^{2}$ A.J. Freeman and C.L. Fu, J. Appl. Phys. 61, 3356 (1987).

${ }^{3}$ S. Blügel, M. Weiner, and P.H. Dederichs, Phys. Rev. Lett. 60, 1077 (1988).

${ }^{4}$ M.T. Kief and W.F. Egelhoff, Jr., Phys. Rev. B 47, 10784 (1993).

${ }^{5}$ M.F. Onellion, C.L. Fu, M.A. Thompson, J.L. Erskine, and A.J. Freeman, Phys. Rev. B 33, 7322 (1986).

${ }^{6}$ V.L. Moruzzi and P.M. Marcus, Phys. Rev. B 38, 1613 (1988).

${ }^{7}$ G. Allen, Surf. Sci. 74, 79 (1978).

${ }^{8}$ F. Meir, D. Pescia, and T. Schreiber, Phys. Rev. Lett. 48, 645 (1982).

${ }^{9}$ L.E. Klebanoff, S.W. Robey, G. Liu, and D.A. Shirley, Phys. Rev. B 32, 1997 (1987).

${ }^{10}$ D.T. Pierce, R.J. Cellota, and J. Unguris, J. Appl. Phys. 73, 6201 (1993).

${ }^{11}$ R. Wiesendanger, H.J. Güntherodt, G. Güntherodt, R.J. Gambino, and R. Ruf, Phys. Rev. Lett. 65, 247 (1990).

${ }^{12}$ E.R. Moog, S.D. Bader, P.A. Montano, G. Zajac, and T.H. Fleisch, Superlatt. Microstruct. 3, 435 (1987).
${ }^{13}$ M.E. Haugen, Qibiao Chen, and M. Onellion, Phys. Rev. B 49, 14028 (1994).

${ }^{14}$ D. Rouyer, C. Krembel, M.C. Hanf, D. Bolmont, and G. Gewinner, Surf. Sci. 307-309, 477 (1994).

${ }^{15}$ D. Rouyer, C. Krembel, M.C. Hanf, J.C. Peruchetti, D. Bolmont, and G. Gewinner, Surf. Sci. 322, 34 (1995).

${ }^{16}$ J. Jandeleit, Y. Gauthier, and M. Wuttig, Surf. Sci. 319, 287 (1994).

${ }^{17}$ R.G.P. van der Kraan and H. van Kempen, Surf. Sci. 338, 19 (1995).

${ }^{18}$ J.C. Girard, Y. Samson, S. Gauthier, S. Rousset, and J. Klein, Surf. Sci. 306, 294 (1994).

${ }^{19}$ J.A. Stroscio, D.T. Pierce, J. Unguris, and R.J. Celotta, J. Vac. Sci. Technol. B 12, 1788 (1994).

${ }^{20}$ M.-G. Barthès and A. Rolland, Thin Solid Films 76, 45 (1981).

${ }^{21}$ C. Günther, S. Günther, E. Kopatzki, R.Q. Hwang, J. Schróder, J. Vrijmoeth, and R.J. Behm, Ber. Bunsenges. Phys. Chem. 97, 522 (1993).

${ }^{22}$ G.S. Bales and D.C. Chrzan, Phys. Rev. Lett. 74, 4879 (1995).

${ }^{23}$ C. Krembel, M.C. Hanf, D. Bolmont, and G. Gewinner, J. Magn. Magn. Mater. 119, 115 (1993).

${ }^{24}$ Y.-L. He and G.-C. Wang, Phys. Rev. Lett. 71, 3834 (1993). 